\title{
Inhibitory Effects of Stilbenes on the Growth of Three Soybean Pathogens in Culture
}

\author{
Anatoliy V. Lygin, Curtis B. Hill, Michelle Pawlowski, Olga V. Zernova, \\ Jack M.Widholm, Glen L. Hartman, and Vera V. Lozovaya
}

All authors: Department of Crop Sciences, University of Illinois, 1201 W. Gregory Drive, Urbana 61801; and sixth author: United States Department of Agriculture-Agricultural Research Service, University of Illinois, 1101 W. Peabody Drive, Urbana 61801. Accepted for publication 24 January 2014.

\section{ABSTRACT}

Lygin, A. V., Hill, C. B., Pawlowski, M., Zernova, O. V., Widholm, J. M., Hartman, G. L., and Lozovaya, V. V. 2014. Inhibitory effects of stilbenes on the growth of three soybean pathogens in culture. Phytopathology 104:843-850.

The effects of resveratrol and pterostilbene on in vitro growth of three soybean pathogens were tested to determine whether these stilbenic compounds could potentially be targets to increase innate resistance in transgenic soybean plants. Growth of Macrophomina phaseolina, Rhizoctonia solani, and Sclerotinia sclerotiorum was measured on solid and in liquid media amended with resveratrol and pterostilbene (concentration in the media of resveratrol at $100 \mu \mathrm{g} / \mathrm{ml}$ and pterostilbene at $25 \mu \mathrm{g} / \mathrm{ml}$ ). All three fungi were very sensitive to pterostilbene in potato dextrose agar (PDA), which reduced colony area of each of the three pathogens to less than half of the control 3 days after incubation. The three fungal pathogens were less sensitive to resveratrol compared with pterostilbene; however, area under the curve (AUC) calculated from colony areas measured over 3 days was significantly $(P<0.05)$ less than the control for $S$. sclerotiorum and $R$. solani on PDA with resveratrol or pterostilbene. AUC for M. phaseolina on PDA with pterostilbene was significantly $(P<0.05)$ lower than the control whereas, on PDA with resveratrol, AUC for M. phaseolina was lower than the control but the difference was nonsignificant $(P>0.05)$. AUC for all three fungi was significantly lower $(P<0.05)$ on PDA with pterostilbene than with resveratrol. In potato dextrose broth (PDB) shake cultures, AUC for all three fungi was significantly $(P<0.01)$ lower in pterostilbene than in the control. AUC for $R$. solani and $S$. sclerotiorum was significantly lower $(P<0.01)$ in resveratrol than the control, whereas AUC for M. phaseolina in resveratrol was lower, but not significantly $(P>0.05)$ different from the control. AUC in pterostilbene was highly significantly $(P<0.01)$ lower than in resveratrol for $M$. phaseolina and significantly $(P<0.05)$ lower for $R$. solani but the difference for $S$. sclerotiorum was nonsignificant $(P>$ $0.05)$. There was a trend for lower mass accumulation of all three fungi in either pterostilbene or resveratrol compared with the control during the course of the experiment; however, S. sclerotiorum appeared to recover from the effects of pterostilbene between days 2 and 4 . Results of biochemical analyses of the PDB over time indicated that the three fungi degraded resveratrol, with nearly $75 \%$ reduction in concentration in M. phaseolina, $80 \%$ in S. sclerotiorum, and $60 \%$ in R. solani PDB cultures by day 4 of fungal growth. M. phaseolina and $S$. sclerotiorum were able to resume growth after early inhibition by resveratrol after its concentration was reduced in the cultures through degradation, whereas $R$. solani was less efficient in resveratrol degradation and was not able to overcome its inhibitory effects on growth. The capacity to degrade pterostilbene was lowest in M. phaseolina compared with S. sclerotiorum and $R$. solani and the recovery of $M$. phaseolina cultures after initial growth inhibition by pterostilbene was minimal. The potential products of resveratrol and pterostilbene degradation by fungi were identified to be dimers and various oxidation products.
Plant diseases pose a major threat to crop production worldwide. The economic importance of plant diseases varies among crops, between geographic areas, and from season to season, due to a multitude of factors. In soybean (Glycine max L. Merr.), worldwide economic losses due to diseases overall was estimated to be $\approx 11 \%$ of total grain production (17). The use of fungicides and nematicides along with host resistance are the primary management options to control soybean diseases.

To withstand and survive stresses produced by different biotic constraints, different resistance mechanisms have evolved in plants. Genetic resistance is the most sustainable option to manage crop diseases when deployed using strategies that minimize selection of virulent pathogen populations. Resistance controlled by simply inherited major resistance genes with major effects on disease development has been effectively utilized to manage many diseases of most crops (31); however, there are many diseases where host resistance is not an available option. Host-

Corresponding author: V. V. Lozovaya; E-mail address: lozovaya@uiuc.edu

http://dx.doi.org/10.1094/PHYTO-10-13-0287-R

(c) 2014 The American Phytopathological Society pathogen interactions not involving a gene-for-gene relationship usually include unspecialized or nonhost-specific facultative parasites or saprophytes inducing a partial resistance response controlled by quantitative trait loci (QTL), with minor effects that limit pathogen colonization and plant damage $(4,32,41,44)$. This kind of plant resistance involves basal or innate resistance in plants and probably interacts with all invading organisms to resist attack in varying degrees, depending on the level of incompatibility between host and pathogen and also on the abilities of the organisms to defeat the innate defenses. Innate resistance response in soybean may be induced by generalist pathogens such as those that cause charcoal rot (Macrophomina phaseolina), Rhizoctonia root rot (Rhizoctonia solani), and Sclerotinia stem rot (Sclerotinia sclerotiorum). Partial resistance to Sclerotinia stem rot controlled by QTL with minor effects on disease development has been reported $(4,32,44)$. This type of resistance usually has low heritability (42) and, thus, it is more difficult to introduce into elite germplasm than resistance controlled by major genes. Few soybean cultivars with partial resistance to these three diseases are available. Genetic engineering to improve the innate or basal resistance in soybean holds promise in offering new sources of resistance to generalist pathogens such as those that cause these soybean diseases (14). 
One essential part of plant innate or basal resistance is the constitutive or induced production of secondary metabolites, phytoanticipins or phytoalexins, correspondingly, which are toxic to many plant pathogens and play an important role in plant interactions with a variety of beneficial and detrimental organisms $(5,38)$. These compounds often provide the first line of plant defense against microbes, not only by killing them but also through slowing pathogen colonization and, thus, allowing other defense mechanisms to be activated by the plant host (3). Phenylpropanoids are known to be involved in soybean-pathogen interactions and can be strongly toxic or inhibitory to pathogens $(18,23,28-30)$. The majority of phytoanticipins or phytoalexins of the family Leguminosae are isoflavonoid derivatives, with pterocarpan being the most abundant isoflavonoid skeleton (9). Implementation of genetic engineering technology in modulation of plant phytoalexin synthesis has the potential to increase disease resistance to a range of pathogens. Reduction or increase of the natural antimicrobial concentrations in plant tissues using genetic modifications has resulted in enhanced or decreased susceptibility to several pathogens $(2,7,11,13,16,21,22,27,33)$.

Different tissues of soybean plants produce the pterocarpan phytoalexin glyceollin in response to attack by pathogens $(6,10$, $12,20,26,28,34)$. We reported the fungicidal effects of the native soybean phytoalexin glyceollin (28) and the involvement of glyceollin in soybean defense responses using in vitro and in vivo tests with different soybean pathogens. The expression of the isoflavone synthase (IFS2) and chalcone synthase (CHS6) transgenes in soybean hairy roots in our previous tests resulted in strong suppression of accumulation of daidzein, the isoflavone precursor of glyceollin. When these roots were inoculated with the soybean pathogens Fusarium virguliforme, M. phaseolina, S. sclerotiorum, and Phytophthora sojae, they produced significantly lower concentrations of induced glyceollin than control hairy roots without the phenolic transgenes (28). Colonization by these pathogens was considerably higher within transformed than control roots. Down regulation of isoflavone synthesis in whole soybean plants or in seed led to strong inhibition of pathogeninducible glyceollin production and increased susceptibility to $M$. phaseolina and $P$. sojae in comparison with nontransgenic control plants (30). These results clearly indicated that the reduced production of the soybean native phytoalexin glyceollin resulted in more severe damage by different pathogens. Therefore, glyceollin was demonstrated to be an important component of the soybean innate defense response. Transgenic expression of nonnative phytoalexins, not normally produced in a plant species, could further increase soybean innate resistance.

Stilbenic compounds have been shown to be effective as antimicrobial, deterrent, or repellent compounds in plants, protecting plants from infection or feeding damage by fungi, bacteria, nematodes, or herbivores $(21,22)$. Stilbenes are relatively simple phenolic compounds synthesized by several plant families, including Vitaceae, Dipterocarpaceae, Gnetaceae, Pinaceae, Fabaceae, Poaceae, Leguminoseae, and Cyperaceae, via the phenyalanine/ polymalonate route, with the last and key enzyme of this pathway being stilbene synthase or resveratrol synthase (STS). Importantly, even though STS enzymes occur naturally in only a few plant species, the substrates for synthesis of stilbenes are present in all higher plants, so that the ability to synthesize stilbenes, the skeleton of which is often based on resveratrol, can be conferred to different plant species by expressing the STS genes $(13,15$, 19,43). Thus, transgenic expression of stilbene synthase from grape (Vitis vinifera) in tobacco (Nicotiana tabacum), tomato (Lycopersicon esculentum), and alfalfa (Medicago sativa) resulted in accumulation of the non-native phytoalexin resveratrol in plant tissues with concomitant increase in resistance to Botrytis cinerea (16), P. infestans (40), and Phoma medicaginis (19), indicating that genetic engineering aimed at non-native phytoalexin production can help to increase innate resistance in plants.
The effects of stilbenic phytoalexins on soybean pathogens have not been studied thus far and the expression of stilbenic phytoalexins or phytoanticipins was not achieved in soybean until recently. The goal of this study was to evaluate the effects of resveratrol and pterostilbene using in vitro tests on the growth of three generalist fungal soybean pathogens: M. phaseolina, $R$. solani (anastomosis group, AG-4), and S. sclerotiorum. M. phaseolina is a pycnidial fungus with worldwide distribution, ubiquitous in most soils, with a wide host range, and causes charcoal rot disease in soybean, $R$. solani is a basidiomycete causing damping off, root rot, and web blight in soybean; and S. sclerotiorum is an ascomycete that can infect most dicots and causes a stem rot disease in soybean (17). These pathogens were selected because they represent different classes of fungi and cause important diseases on soybean in the Midwest.

\section{MATERIALS AND METHODS}

Soybean pathogens. Dr. John Rupe of the University of Arkansas collected the $M$. phaseolina isolate used in this study from soybean in Pinetree, AR. The R. solani isolate was isolated from soybean in Illinois. The $S$. sclerotiorum isolate, 105HT, was collected from soybean in Illinois, and has been used in several previous studies $(25,41,42)$. All three isolates used in this study were maintained on potato dextrose agar (PDA) at the Soybean Pathogen Collection Center at the University of Illinois, Urbana.

Fungal growth on stilbene-amended culture media. Growth on the stilbenes was measured on solid and in liquid media in two-way factorial tests with three fungal species (M. phaseolina, $R$. solani, or S. sclerotiorum) and stilbenes (resveratrol or pterostilbene). Prior to preparation of solid media amended with stilbenes, stock solutions of resveratrol or pterostilbene were prepared by dissolving the compounds in $1 \mathrm{ml}$ of absolute ethanol in amounts to get a final concentration in the media of resveratrol at $100 \mu \mathrm{g} / \mathrm{ml}$ and pterostilbene at $25 \mu \mathrm{g} / \mathrm{ml}$. Then, the ethanol solutions were added to $500 \mathrm{ml}$ of cooled PDA medium before agar solidification. A control treatment was prepared by adding $1 \mathrm{ml}$ of absolute ethanol without either stilbenic compound to the cooled agar medium. Petri dishes $(100 \mathrm{~mm}$ in diameter by $15 \mathrm{~mm}$ deep) containing $20 \mathrm{ml}$ of PDA with and without resveratrol or pterostilbene were inoculated with 8-mm-diameter plugs cut from the margins of actively growing fungal PDA cultures by placing plugs in the center of each petri dish with the mycelium side down in direct contact with the medium. The cultures were incubated at $24^{\circ} \mathrm{C}$ in the dark. Colony diameter (in millimeters) was measured with a clear plastic ruler at the same time of day for three consecutive days (24-h intervals). Colony area was calculated using the area of a circle formula as $\pi(\text { diameter } / 2)^{2}$. Area under the curve (AUC) over 3 days was calculated with the colony area using the midpoint rule method (39). Two tests with different randomizations were completed. The dishes in each test were arranged in a completely randomized design on a laboratory bench, with five replicates for each fungus-stilbene concentration treatment.

When fungi were grown in liquid medium, $100 \mu \mathrm{l}$ of stilbenic compound stock ethanol solution or $100 \mu$ lof absolute ethanol (negative control) were added to $50 \mathrm{ml}$ of potato dextrose broth (PDB) (BD Difco, Sparks, MD) in 125-ml Erlenmeyer flasks to get a final concentration in the media of resveratrol at $100 \mu \mathrm{g} / \mathrm{ml}$ or pterostilbene at $25 \mu \mathrm{g} / \mathrm{ml}$. Each flask was inoculated with a single 10-mm-diameter plug cut from the margin of a 3- to 5-dayold PDA culture, depending on the fungal species growth rate. Inoculated flasks were capped with sterile foam plugs, which were covered with a double layer of aluminum foil, and the flasks were incubated on an orbital shaker at $25^{\circ} \mathrm{C}$ in the laboratory. Due to limited space on the shaker, tests were completed over time, with one fungus and one phytoalexin tested at a time. Two tests with different randomizations were completed for each fungus 
and stilbene. Flasks for each stilbene treatment of each fungus were arranged in a completely randomized design on the shaker. In each test, there were two levels of stilbene (with or without), three sampling days (days 1, 2, and 4 after inoculation), and five replicates for each stilbene level and sampling day, giving a total of 30 flasks. At the beginning of each test, on day 0 , a sample of $500 \mu \mathrm{l}$ of medium was collected from each of the 30 flasks for biochemical analysis. On days 1, 2, and 4 after inoculation, the mycelia were removed from the five replicate flasks of each stilbene treatment (with and without) for the sampling day and dried in an oven at $35^{\circ} \mathrm{C}$. Dried mass (in grams) of mycelia for each flask was weighed on an electronic balance and recorded after the mass was determined to be free of moisture, indicated by stable weights over time. AUC was calculated with the mass data across sampling days 1,2, and 4 using the midpoint rule, as above. A sample of $500 \mu \mathrm{l}$ of medium was collected from each flask for biochemical analysis when the mycelia were removed. Media samples were kept frozen until analysis.

Extraction and measurement of resveratrol and pterostilbene, and products of their fungal degradation. To monitor the levels of stilbenes in amended PDB cultures, the 500- $\mu$ l samples collected from each culture were centrifuged at $12,000 \times g$ for $10 \mathrm{~min}$ prior to high-performance liquid chromatography (HPLC) measurement of stilbene level. In addition, $5 \mathrm{ml}$ from each sample were extracted twice with $3 \mathrm{ml}$ of ethyl acetate; then, extracts were evaporated to dryness on a rotor evaporator under reduced pressure, dissolved in $80 \%$ methanol, and analyzed by HPLC to determine stilbene degradation metabolites.

HPLC separations of resveratrol, pterostilbene, and their metabolites were carried out in a Separation Module 2690 with 960 PDA Detector from Waters (Milford, MA) supplied with a Prevail C18 column (Alltech, Deerfield, IL), 250 by $4.6 \mathrm{~mm}$ by $5 \mu \mathrm{m}$. Solvent A was water-acetonitrile-acetic acid (95:5:0.5) and solvent B was acetonitrile-water-acetic acid (95:5:0.5). The flow rate was $1 \mathrm{ml} / \mathrm{min}$. Elution was done with a linear gradient from 5 to $15 \%$ $\mathrm{B}$ for $10 \mathrm{~min}$, then to $30 \%$ for $15 \mathrm{~min}$, and a linear gradient to $45 \%$ B for $20 \mathrm{~min}$. After that, the column was washed with $95 \%$ acetonitrile for $3 \mathrm{~min}$ and equilibrated at $5 \% \mathrm{~B}$ between runs for $3 \mathrm{~min}$. Total sample-to-sample time was $55 \mathrm{~min}$. Detection was done by UV absorbance at $306 \mathrm{~nm}$ for stilbenes and their derivatives and at $295 \mathrm{~nm}$ for metabolites.

Liquid chromatography mass spectrometry (LC-MS) analysis of resveratrol and pterostilbene metabolites was carried out in Agilent 1100 LC/MSD Trap XCT Plus supplied with the column and using the same HPLC conditions as above, except that the flow rate was $500 \mu \mathrm{l} / \mathrm{min}$. The ion source was electrospray ionization, negative mode, nebulizing gas $=\mathrm{N}^{2}, 350^{\circ} \mathrm{C}, 9 \mathrm{ml} / \mathrm{min}$, and capillary voltage $=3,500 \mathrm{~V}$.

Resveratrol, pterostilbene, and their metabolites were identified using HPLC with PDA detector and LC-MS. The comparison of the sample retention times and UV spectra was done with authentic standards for resveratrol (Sigma-Aldrich, St. Louis, MO), pterostilbene, piceatannol (Cayman Chemical, Ann Arbor, MI), p-hydroxy benzoic acid, p-hydroxybezaldehyde, 3,4-dihydroxybenzoic acid, 3,5-dihydroxybenzoive acid, 3.5-dihydroxybenzaldehyde, 3,5-dimethoxybenzoic acid, and 3,5-dimethoxybenzaldehyde (Sigma-Aldrich).

Statistical analyses. All statistical analyses were performed with JMP (version 10; SAS Institute, Cary, NC). Data from each of the repeated tests of growth on PDA and PDB amended with stilbenes were combined when analyses for variance heterogeneity between the tests were nonsignificant $(P<0.05)$. Analysis of variance was performed on AUC data collected from the PDA and PDB tests to test the effects of fungus and stilbene on fungal growth in the solid or liquid media. Single degree-of-freedom comparisons between the phytoalexins and controls and between the phytoalexins were performed using contrasts to determine the significance of the differences of the effects on the growth of each of the three fungi between the treatments. Least square means and standard errors of the colony area measured in the PDA tests and the mycelial mass (in milligrams) measured in the PDB tests for each fungus and phytoalexin combination over days of incubation were computed using JMP and are presented in the figures.

\section{RESULTS AND DISCUSSION}

Fungicidal effects of resveratrol and pterostilbene. We compared growth of soybean pathogens $M$. phaseolina, $R$. solani, and $S$. sclerotiorum on resveratrol- or pterostilbene-amended PDA with growth on medium without stilbenic phytoalexins. We also used the stilbene-amended medium not inoculated with fungi as an additional control.

Heterogeneity between all of the repeated solid and liquid media tests was nonsignificant $(P>0.05)$. Therefore, the data of the repeated tests were combined for analysis.

Fungal growth on agar medium. There was a highly significant $(P<0.001)$ fungus-stilbene interaction for AUC calculated from the colony area (in square centimeters) over the 3 days of the PDA test. Single degree-of-freedom comparisons between the phytoalexins and controls and between the phytoalexins indicated that the differences in AUC between media with resveratrol and the control were highly significant $(P<0.001)$ for $S$. sclerotiorum and $R$. solani but not for $M$. phaseolina. All differences between media with pterostilbene and the control and between resveratrol and pterostilbene were highly significant $(P<$ 0.001 ) for all three fungi. Colony area for $M$. phaseolina on PDA amended with resveratrol at $100 \mu \mathrm{g} / \mathrm{ml}$ was consistently lower than the control (Fig. 1A). Colony area was lower than the control 2 and 3 days after inoculation for $S$. sclerotiorum and at 3 days for $R$. solani on PDA amended with resveratrol at $100 \mu \mathrm{g} / \mathrm{ml}$. Colony area of all three fungi grown on PDA amended with pterostilbene at $25 \mu \mathrm{g} / \mathrm{ml}$ was lower than on the control plates at days 2 and 3 (Fig. 1).

Results indicated that the three fungi were much less sensitive to resveratrol compared with pterostilbene (Fig. 1). Concentrations of soybean native glyceollins in the range $(24$ to $47 \mu \mathrm{g} / \mathrm{ml})$ used for pterostilbene in this study resulted in fungicidal effects on fungal growth (28) comparable to those found for pterostilbene in this study, although pterostilbene suppressed fungal growth to much higher extent. These results also indicated that much higher concentrations of resveratrol would be required to suppress fungal colony growth to the extent that pterostilbene did.

Mycelial mass accumulation in liquid medium. As found for the PDA tests, the heterogeneity between repeated PDB tests was nonsignificant $(P>0.05)$ and the data were combined prior to analysis. There was a significant $(P<0.05)$ fungus-stilbene interaction for AUC. Single degree-of-freedom comparisons between the phytoalexins and controls and between the phytoalexins indicated that the differences in AUC between media with resveratrol and the control were highly significant $(P<0.001)$ for $S$. sclerotiorum and $R$. solani but there was no difference between AUC in resveratrol or the control for M. phaseolina, which was consistent with what was found in PDA above. AUC in pterostilbene was highly significantly $(P<0.001)$ lower from the control for all three fungi. AUC in resveratrol was highly significantly different $(P<0.001)$ from pterostilbene for $M$. phaseolina and $R$. solani but the difference in AUC between the stilbenes for $S$. sclerotiorum was nonsignificant $(P>0.05)$.

For $M$. phaseolina, the mycelial mass (in milligrams) in PDB with resveratrol $(100 \mu \mathrm{g} / \mathrm{ml})$ was consistently lower than the control throughout the experiment (Fig. 2A), whereas M. phaseolina mycelial mass on days 2 and 4 was lower than the control in the medium with pterostilbene $(25 \mu \mathrm{g} / \mathrm{ml})$ (Fig. 2B), appearing to have a flat growth rate throughout the test. Mycelial mass of $S$. sclerotiorum was lower in PDB amended with resveratrol on days 2 and 4 (Fig. 2C). S. sclerotiorum mass was lower in PDB 
amended with pterostilbene at day 2 but not at day 4 (Fig. 2D), appearing to suggest that this fungus was able to overcome the growth inhibitory effects of the stilbene between those two sampling days. $R$. solani mycelial mass in PDB amended with resveratrol was lower than the control at day 4 , with a flat growth rate throughout the experiment in that stilbene (Fig. 2E). In PDB amended with pterostilbene, $R$. solani mycelial mass was lower on day 2 and much lower on day 4 than the control (Fig. 2F). Overall results indicated that the resveratrol treatment had the largest effect on $R$. solani mycelial growth and the smallest effect on $M$. phaseolina. Mycelial growth of $R$. solani and M. phaseolina was also suppressed by pterostilbene, with $M$. phaseolina more sensitive than $R$. solani, whereas $S$. sclerotiorum mycelial growth appeared to only be suppressed up to day 2 , after which it grew rapidly.

These results showed that the stilbenic compounds resveratrol and pterostilbene had the ability to suppress the growth of three soybean fungal pathogens in vitro to different degrees, depending
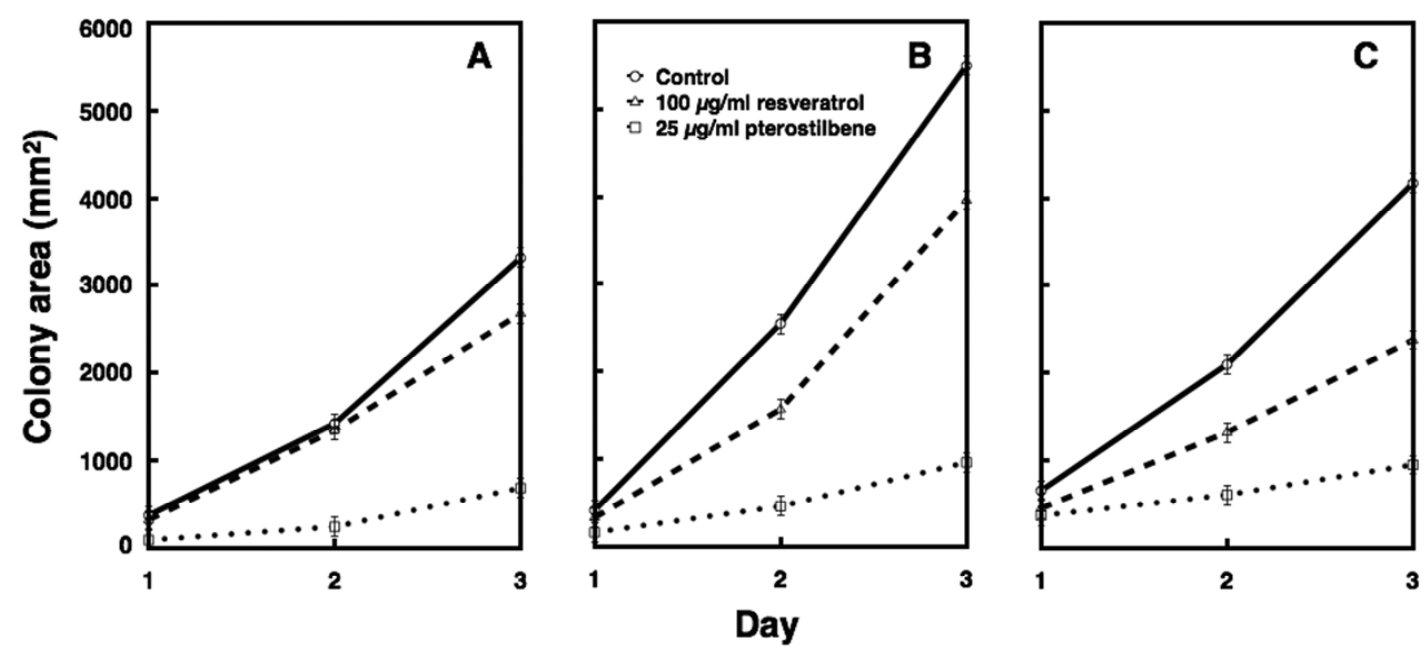

Fig. 1. Growth of three soybean fungal pathogens-A, Macrophomina phaseolina; B, Sclerotinia sclerotiorum; and C, Rhizoctonia solani-over 3 days after inoculation on potato dextrose agar medium amended with different levels of stilbenes incubated at $25^{\circ} \mathrm{C}$. Error bars $\pm 117 \mathrm{~mm}$ (standard error).

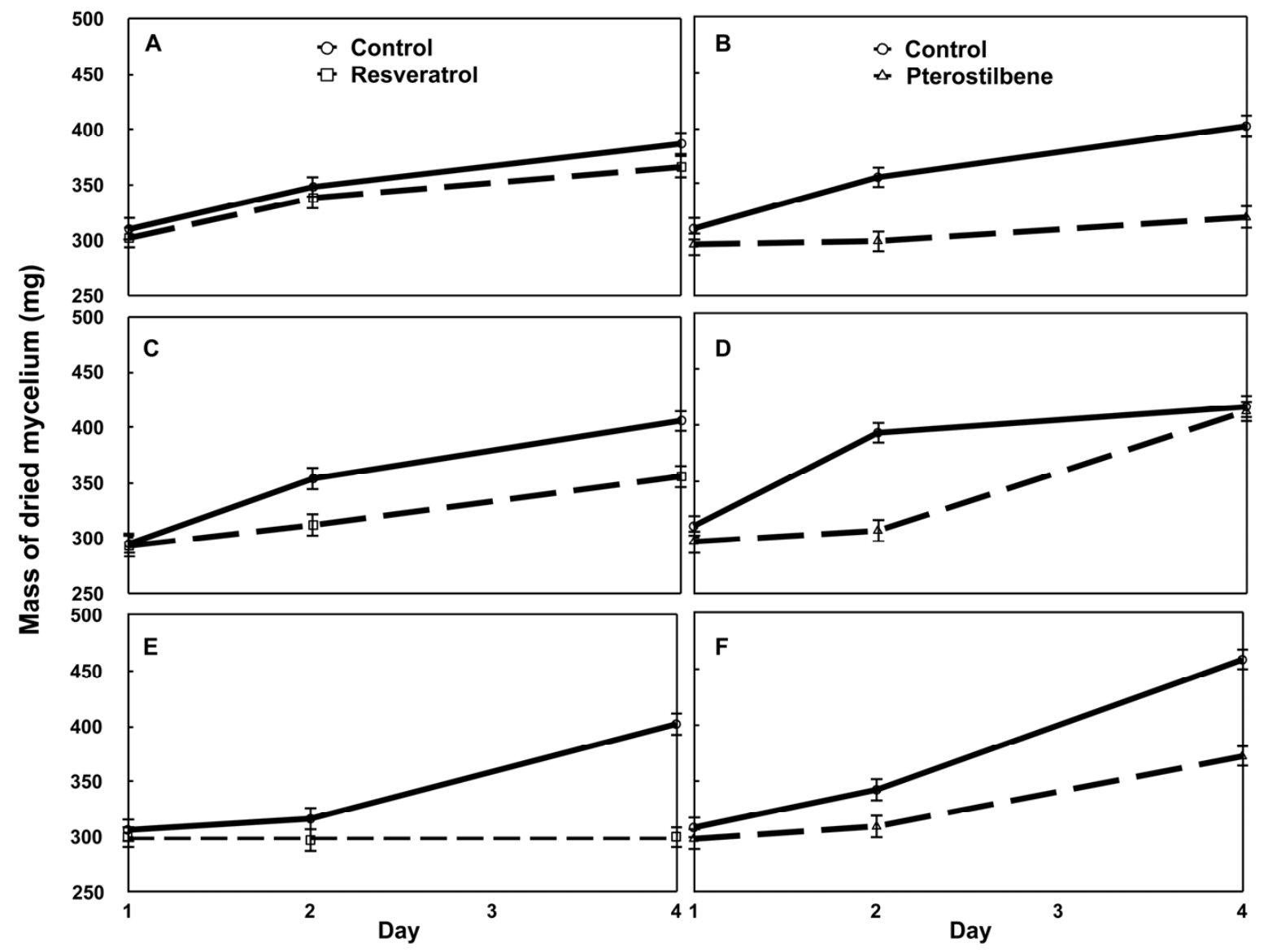

Fig. 2. Growth of three soybean fungal pathogens- $\mathbf{A}$ and $\mathbf{B}$, Macrophomina phaseolina; $\mathbf{C}$ and $\mathbf{D}$, Sclerotinia sclerotiorum; and $\mathbf{E}$ and $\mathbf{F}$, Rhizoctonia solani-in $50 \mathrm{ml}$ of potato dextrose broth amended with $\mathbf{A}, \mathbf{C}$, and $\mathbf{E}$, resveratrol at $100 \mu \mathrm{g} / \mathrm{ml}$ or $\mathbf{B}, \mathbf{D}$, and F, pterostilbene at $25 \mu \mathrm{g} / \mathrm{ml}$. Error bars $\pm 10 \mathrm{mg}$ (standard error). Mass was measured on days 1,2, and 4 only. 
on the stilbene and the fungus. It is expected that the expression of resveratrol and pterostilbene at the levels used in these experiments, which clearly suppressed fungal growth in vitro, would enhance soybean innate resistance against these pathogens in vivo. Work is in progress to test the effects of these stilbenes synthesized in planta on pathogen colonization of plant tissues.

Degradation of resveratrol and pterostilbene. The ability of these select soybean fungal pathogens to metabolize resveratrol and pterostilbene, compounds that are not normally produced by soybean plants, was tested by measuring resveratrol and pterostilbene levels in the stilbene-amended PDB cultures over time. Essential metabolization or degradation of resveratrol (used in concentration of $100 \mu \mathrm{g} / \mathrm{ml}$ ) and pterostilbene (used in concentration of $25 \mu \mathrm{g} / \mathrm{ml}$ ) by all three fungi tested was detected. On day 4 in PDB-amended with resveratrol, we found only $\approx 25 \%$ of its initial concentration in M. phaseolina samples, $20 \%$ in S. sclerotiorum samples, and $40 \%$ in $R$. solani samples (Fig. 3A to C). We can assume that, after initial suppression of $M$. phaseolina and $S$. sclerotiorum growth by resveratrol, these fungi were able to metabolize a portion of this phytoalexin in the medium and then resumed growth after resveratrol concentration was lowered to apparent non-growth-suppressive levels, even though their mycelial mass accumulation at day 4 was less than the mycelial mass grown without resveratrol. $R$. solani was less efficient in degradation and more sensitive to resveratrol, and probably was not able to overcome its growth inhibitory effects (Figs. 2 and 3). The capacity to metabolize or degrade pterostilbene in liquid medium was lowest in $M$. phaseolina compared with S. sclerotiorum and $R$. solani (Fig. 4C) and the recovery after initial growth suppression caused by pterostilbene was minimal in M. phaseolina cultures (Figs. 2 and 4).

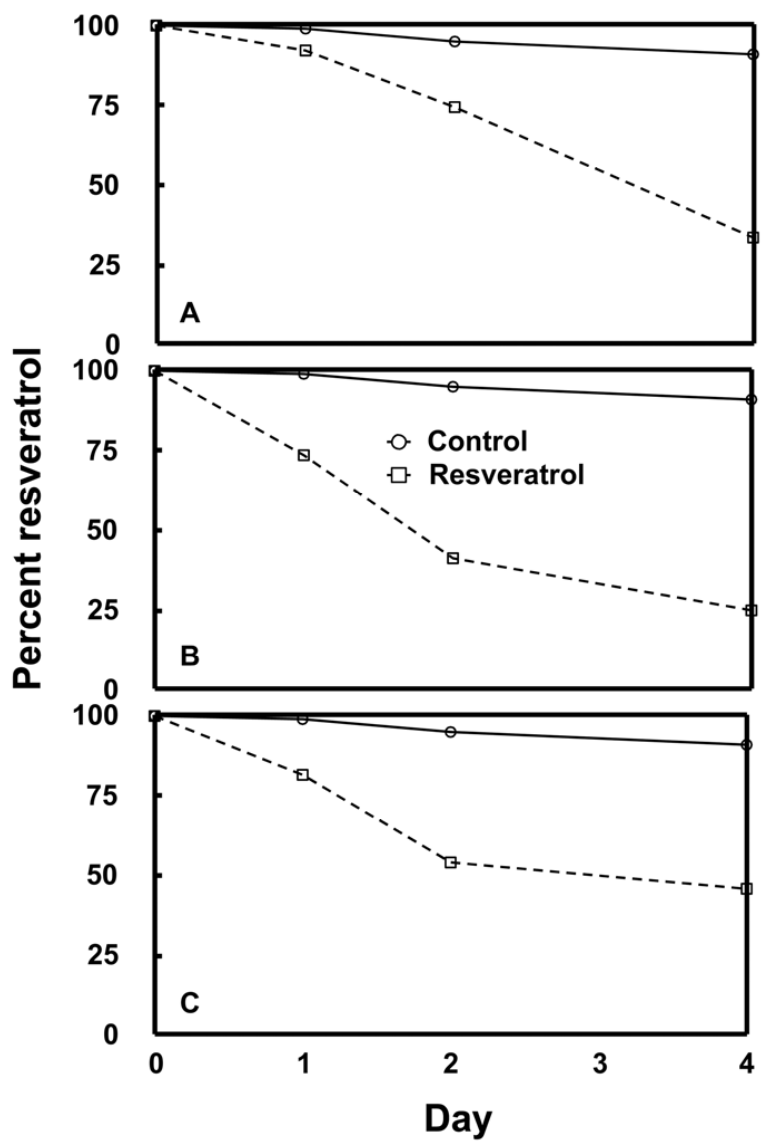

Fig. 3. Percent reduction of resveratrol concentration in potato dextrose brothamended cultures of three soybean fungal pathogens over 4 days during incubation at $25^{\circ} \mathrm{C}$. A, Macrophomina phaseolina; B, Sclerotinia sclerotiorum; and $\mathbf{C}$, Rhizoctonia solani.
Identification of products of resveratrol and pterostilbene degradation. HPLC analysis of ethyl acetate extracts from the $M$. phaseolina and $S$. sclerotiorum PDB growth medium, amended with resveratrol and obtained after day 2 and 4, showed the presence of p-hydroxybenzoic acid (retention time $\left[R_{t}\right]=$ $5.9 \mathrm{~min})$, p-hydroxybenzaldehyde $\left(R_{t}=8.1 \mathrm{~min}\right), 3,5$-dihydroxybenzoic acid $\left(R_{t}=9.2 \mathrm{~min}\right)$, 3,5-dihydroxybenzaldehyde $\left(R_{t}=\right.$ $12.6 \mathrm{~min})$, and also of 3.4-dihydroxybenzoic acid $\left(R_{t}=9.8 \mathrm{~min}\right)$ as products of resveratrol conversion by these two fungi. The same resveratrol metabolization products, with the exception of 3,4-dihydrobenzoic acid, were found in $R$. solani PDB growth cultures amended with resveratrol. Piceatannol or $3.4,3^{\prime} 5^{\prime}$ tetrahydroxystilbene $\left(R_{t}=17.5 \mathrm{~min}\right),[\mathrm{M}-\mathrm{H}]^{-}$at $m / z 243$ was detected in the growth medium extract on day 2 of M. phaseolina and $S$. sclerotiorum incubation with resveratrol. In addition, several compounds with retention times of 21.6, 23.0, 28.4, and $36.2 \mathrm{~min}$ and UV spectra were also found that were very similar to that of resveratrol. Interestingly, compounds with $R_{t}=21.6$ and 23.0 were present only in the $M$. phaseolina and $S$. sclerotiorum cultures, respectively, whereas the compounds with higher retention times were found in samples of all three fungi. LC-MS analysis of these unknown peaks showed that molecular masses of two of the former compounds were $[\mathrm{M}-\mathrm{H}]^{-}$at $m / z \quad 471$ and molecular masses of two latter compounds were $[\mathrm{M}-\mathrm{H}]^{-}$at $m / z$ 453. We assigned the structure of these metabolites as oxidimers and dimers of resveratrol. Based on our chemical analyses, we concluded that degradation of resveratrol by M. phaseolina and $S$. sclerotiorum could occur through the potential reactions presented in Figure 5, with reaction $1=$ oxidative cleavage of resveratrol double bond with production of benzaldehydes and benzoic acids; reaction 2 = hydroxylaton of resveratrol at position

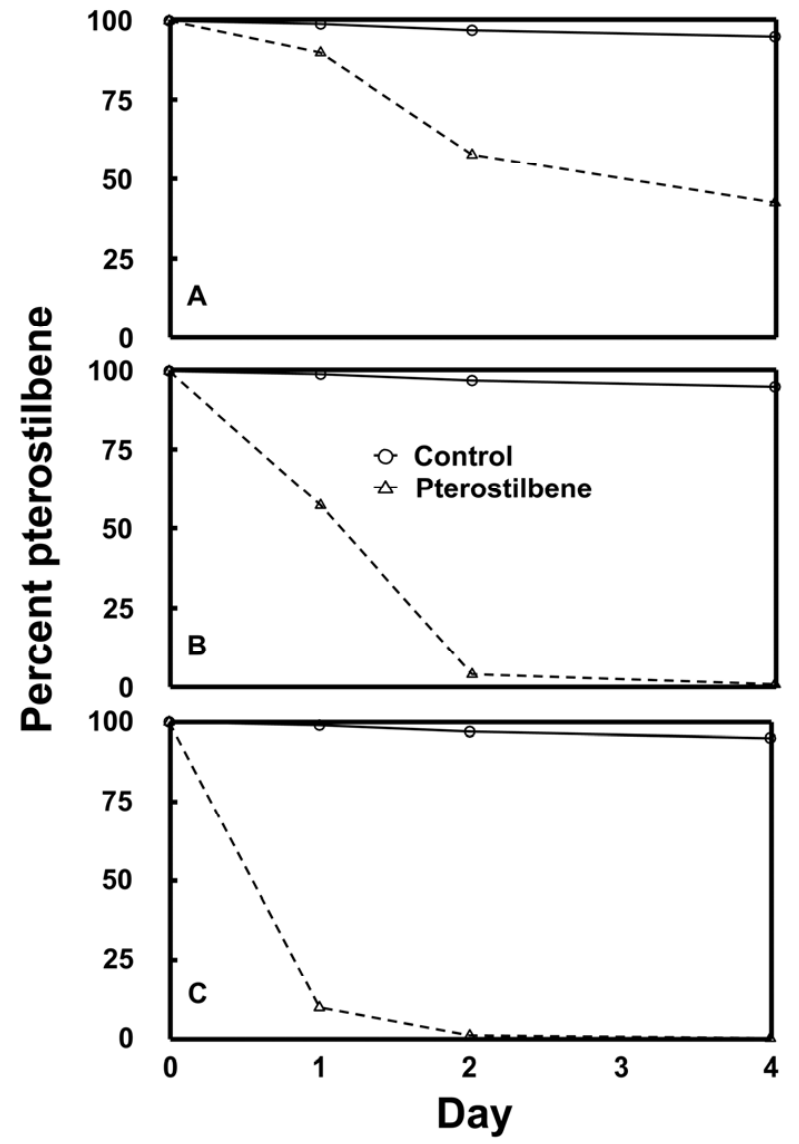

Fig. 4. Percent reduction of pterostilbene concentration in potato dextrose broth-amended cultures of three soybean fungal pathogens over 4 days during incubation at $25^{\circ} \mathrm{C}$. A, Macrophomina phaseolina; $\mathbf{B}$, Sclerotinia sclerotiorum; and $\mathbf{C}$, Rhizoctonia solani. 
3 with production of piceatannol, followed by its oxidation (reaction 3); reaction $4=$ dimerization of resveratrol; and reaction $5=$ dimerization of piceatannol with resveratrol.

Production of resveratrol dimers by $B$. cinerea from resveratrol was reported by Chichewicz et al. (8). Ability to produce piceatannol from resveratrol by bacterial cytochrome P450 was demon- strated by Kim et al. (24). Based on our results, $R$. solani appears to lack the enzyme for resveratrol hydroxylation, making it unable to form piceatannol, at least in detectable amounts, or it may rapidly convert it to other metabolites. It is not known whether other basidiomycetes also lack the enzyme for resveratrol hydroxylation.

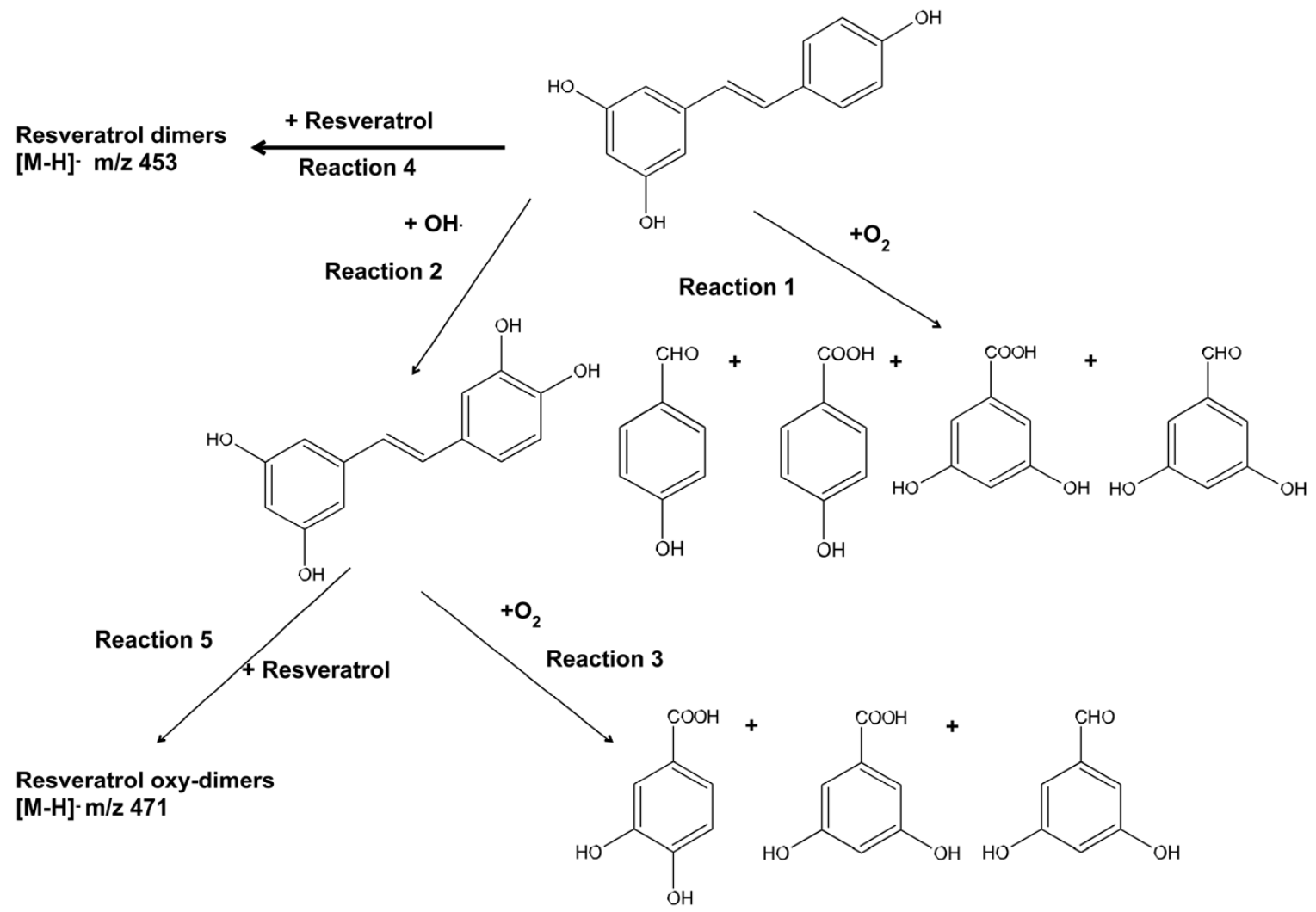

Fig. 5. Possible reactions of resveratrol degradation by Sclerotinia sclerotiorum and Macrophomina phaseolina.<smiles>COc1cc(/C=C/c2ccc(O)c(O)c2)cc(OC)c1</smiles>

Fig. 6. Possible reactions of pterostilbene degradation by Sclerotinia sclerotiorum and Macrophomina phaseolina. 
The levels of all resveratrol metabolites identified in this present study did not increase over longer incubation time but decreased by 4 days of fungal growth, indicating that these metabolites were not the final products of resveratrol degradation, and compounds with simpler and more oxidized structures could be formed during further conversion. These compounds could have been missed with ethyl acetate extraction. Microscopic observations indicated that resveratrol oxidation, specifically linked to a laccase-like polyphenol-oxidase, could occur in the cytoplasm of the $B$. cinerea conidia and the resulting products later accumulate in the vacuole (1).

When $M$. phaseolina and $S$. sclerotiorum were incubated in pterostilbene-amended PDB, 3,5-dimethoxybenzoic acid $\left(R_{t}=\right.$ $38.0 \mathrm{~min})$ and 3,5-dimethoxybenzaldehyde $\left(R_{t}=41.4 \mathrm{~min}\right)$ were also found in addition to p-hydrobenzaldehyde, p-hydrobenzoic acid, and 3,4-dihydroxybenzoic acid, as well as several unknown peaks, with $R_{t}=42.5 \mathrm{~min}, \lambda_{\max } 324 \mathrm{~nm} ; R_{t}=35.0 \mathrm{~min}, \lambda_{\max } 306$ and $319 \mathrm{~nm} ; R_{t}=46.5$ and $48.2 \mathrm{~min}, \lambda_{\max } 307$ shoulder curvechanging slope without peak appearance and $319 \mathrm{~nm}$. LC-MS analysis revealed that a compound with $R_{t}=42.5 \mathrm{~min}$ gave a molecular ion $[\mathrm{M}-\mathrm{H}]^{-}$at $\mathrm{m} / \mathrm{z}, 271$ and had the same UV spectrum as $3,4,3^{\prime}, 5^{\prime}$-tetrahydroxy stilbene, which allowed us to assign the structure as 3,4 ,-dihydroxy- $3^{\prime}, 5^{\prime}$-dimethoxy stilbene. Other peaks had the masses of molecular ions $[\mathrm{M}-\mathrm{H}]^{-}$at $\mathrm{m} / z, 497$ for compounds with $R_{t}=35.0 \mathrm{~min}$ and $\mathrm{m} / \mathrm{z} 481$ for compounds with $R_{t}=$ 46.5 and $482 \mathrm{~min}$. We assume that the former compound is the adduct of 3,4,-dihydroxy-3', $5^{\prime}$-dimethoxy stilbene and pterostolbene and two other compounds are pterostilbene dimers. We did not find 3,4,-dihydroxy-3',5'-dimethoxy stilbene and its adduct with pterostilbene in $R$. solani cultures. Based on these results, we believe that the mechanism of pterostilbene degradation is similar to that of resveratrol (Fig. 6).

We measured each stilbenic compound concentration over time in the absence of fungus and the data are presented in Figures 5 and 6. In the control, only insignificant decrease of both compound concentrations was found in liquid medium without fungus after 4 days of incubation (in the range of 7 to $9 \%$ for resveratrol and 4 to $5 \%$ for pterostilbene). The resveratrol and pterostilbene conversion metabolites described above were not detected in resveratrol- and pterostilbene-amended medium without fungi, indicating that they are products of fungaldegrading activity, and only the presence of stilbene cis-isomers was found in these control samples, indicating the possibility of stilbene isomerization in medium over time (conversion of some stilbene trans forms to cis forms). The effects of resveratrol and pterostilbene derivatives, resulting from degrading activity on fungal growth, still need to be tested.

It was stated earlier that the formation of resveratrol dimers could be the initial step in resveratrol detoxification by the fungal grapevine pathogen $B$. cinerea (8). In that study, none of the resveratrol transformation compounds produced by $B$. cinerea showed antiviral activity.

Results of our study suggest that expression of resveratrol and pterostilbene synthesis in soybean tissues could potentially increase soybean plant innate resistance and slow or suppress the development of soybean pathogens. Because most pathogens can adapt to major resistance genes and overcome resistance, rapid accumulation of non-native phytoalexins during infection could help increase the durability of major gene resistance, and could also help stabilize soybean grain production in the presence of diseases. Therefore, engineering soybean to enable the crop to produce non-native phytoalexins, and enhancing the innate defense response against pathogen invasion or the capacity to prevent phytoalexin degradation by pathogens, would be a suitable sustainable strategy for managing pathogens $(35,36)$, for which effective genetic resistance is lacking, such as Sclerotinia stem rot or charcoal rot. In addition, identification of enzymes involved in phytoalexin degradation or detoxification will improve our under- standing on how pathogens defeat the phytoalexins. With this knowledge, it will be possible to design antifungal agents selective against specific pathogens $(37,38)$ or clone fungal genes controlling phytoalexin-detoxifying enzymes to use to silence these genes through plant transformation.

\section{LITERATURE CITED}

1. Adrian, M., Rajaei, H., Jeandet, P., Veneau, J., and Bessis, R. 1998. Resveratrol oxidation in Botrytis cinerea conidia. Phytopathology 88:472476.

2. Aharoni, A., and Galili, G. 2011. Metabolic engineering of the plant primary-secondary metabolism interface. Curr. Opin. Biotechnol. 22:239244.

3. Ahuja, I., Kissen, R., and Bones, A. M. 2012. Phytoalexins in defense against pathogens. Trends Plant Sci. 17:73-90.

4. Bariana, H. S., Bansal, U. K., Basandrai, D., and Chhetri, M. 2013. Disease resistance. Pages 291-314 in: Genomics and Breeding for Climate-Resilient Crops, Volume 2. Target Traits. C. Kole, ed. Springer, Berlin, Heidelberg.

5. Bednarek, P., and Osbourn, A. 2009. Plant-microbe interactions: chemical diversity in plant defense. Science 324:746-748.

6. Bhattacharyya, M. K., and Ward, E. W. B. 1985. Differential sensitivity of Phytophthora megasperma f sp. glycinea to glyceollin isomers. Physiol. Mol. Plant. Pathol. 27:299-310.

7. Campbell, M. A., Fitzgerald, H. A., and Ronald, P. C. 2002. Engineering pathogen resistance in crop plants. Transgenic Res. 11:599-613.

8. Chichewicz, R. H., Kouzi, S. A., and Hamann, M. T. 2000. Dimerization of resveratrol by the grapevine pathogen Botrytis cinerea. J. Nat. Prod. 63:29-33.

9. Dixon, R. A. 1999. Isoflavonoids: biochemistry, molecular biology, and biological functions. Pages 773-823 on: Comprehensive Natural Products Chemistry, Vol 1. Polyketides and Other Secondary Metabolites Including Fatty Acids and Their Derivatives. U. Sankawa, ed. Elsevier, Oxford.

10. Ebel, J., and Grisebach, H. 1988. Defense strategies of soybean against the fungus Phytophthora megasperma f. sp. glycinea: a molecular analysis. Trends Biochem. Sci. 13:23-27.

11. Essenberg, M. 2001. Prospects for strengthening plant defenses through phytoalexin engineering. Physiol. Mol. Plant Pathol. 59:71-81.

12. Graham, T. L., and Graham, M. Y. 1991. Glyceollin elicitors induce major but distinctly different shifts in isoflavonoid metabolism in proximal and distal soybean cell populations. Mol. Plant-Microbe Interact. 4:60-68.

13. Grobkinsky, D. K., van der Graaff, E., and Roitsch, T. 2012. Phytoalexin transgenics in crop protection-fairy tale with a happy end? Plant Sci. 195:54-70.

14. Gurr, S. J., and Rushton, P. J. 2005. Engineering plants with increased disease resistance: what are we going to express? Trends Biotechnol. 23:275-282.

15. Hain, R., Bieseer, B., Kindl, H., Schroeder, G., and Stoecker, R. 1990. Expression of a stilbene synthase gene in Nicotiana-tabacum results in synthesis of the phytoalexin resveratrol. Plant Mol. Biol. 15:325-336.

16. Hain, R., Reif, H. J., Krause, E., Langebartels, R., Kindl, H., Vornam, B., Wiese, W., Schmelzer, E., Schreier, P. H., Stocker, R. H., and Strenzel, K. 1993. Disease resistance results from foreign phytoalexin expression in a novel plant. Nature 361:153-156.

17. Hartman, G. L., Sinclair, J. B., and Rupe, J. C., eds. 1999. Compendium of Soybean Diseases, 4th ed. American Phytopathological Society Press, St. Paul, MN.

18. Hill, C. B., Bowen, C. R., and Hartman, G. L. 2013. Effect of fungicide application and cultivar on soybean green stem disorder. Plant Dis. 97:1212-1220.

19. Hipskind, J. D., and Paiva, N. L. 2000. Constitutive accumulation of a resveratrol-glucoside in transgenic alfalfa increases resistance to Phoma medicaginis. Mol. Plant-Microbe Interact. 13:551-562.

20. Huang, J.-S., and Barker, K. R. 1991. Glyceollin I in soybean-cyst nematode interactions. Plant Physiol. 96:1302-1307.

21. Jeandet, P., Delaunois, B., Conreux, A., Donnez, D., Nuzzo, V., Cordelier, S., Clement, C., and Courot, E. 2010. Biosynthesis, metabolism, molecular engineering and biological functions of stilbene phytoalexins in plants. Biofactors 36:331-341.

22. Jeandet, P., Douillet, A. C., Debord, S., Sbaghi, M., Bessis, R., and Adrian, M. 2002. Phytoalexins from the Vitaceae: biosynthesis, phytoalexin gene expression in transgenic plants, antifungal activity, and metabolism. J. Agric. Food Chem. 50:2731-2741.

23. Jiang, Y. N., Haudenshield, J. S., and Hartman G. L. 2012. Response of soybean fungal and oomycete pathogens to apigenin and genistein. Mycology 3:153-157.

24. Kim, D.-H., Ahn, T., Jung H.-C., Pan J.-G., and Yun, C-H. 2009. Generation of the human metabolite piceatannol from the anticancer- 
preventive agent resveratrol by bacterial cytochrome P450 BM3. Drug Metab. Dispos. 37:932-936.

25. Kull, L. S., Vuong, T. D., Powers, K. S., Eskridge, K. M., Steadman, J. R., and Hartman, G. L. 2003. Evaluation of resistance screening methods for Sclerotinia stem rot of soybean and dry bean. Plant Dis. 87:1471-1476.

26. Lozovaya, V. V., Lygin, A. V., Li, S., Hartman, G. L., and Widholm, J. M. 2004. Biochemical response of soybean roots to Fusarium solani f. sp. glycines infection. Crop Sci. 44:819-826.

27. Lozovaya, V. V., Lygin, A. V., Zernova, O. V., and Widholm, J. M. 2005. Genetic engineering of plant disease resistance by modification of the phenylpropanoid pathway. Plant Biosyst. 139:20-23.

28. Lygin, A. V., Hill, C. B., Zernova, O. V., Crull, L., Widholm, J. M., Hartman, G. L., and Lozovaya, V. V. 2010. Response of soybean pathogens to glyceollin. Phytopathology 100:897-903.

29. Lygin, A. V., Li, S., Vittal, M., Widholm, J. M., Hartman, G. L., and Lozovaya, V. V. 2009. Response of soybean pathogens to glyceollin. Phytopathology 99:1412-1420.

30. Lygin, A. V., Zernova, O. V., Hill, C. B., Kholina, N. A., Widholm, J. M., Hartman, G. L., and Lozovaya. V. V. 2013. Glyceollin is an important component of soybean plant defense against Phytophthora sojae and Macrophomina phaseolina. Phytopathology 103:984-994.

31. Michelmore, R. W., Christopoulou, M., and Caldwell, K. S. 2013. Impacts of resistance gene genetics, function, and evolution on a durable future. Annu. Rev. Phytopathol. 51:291-319.

32. Miklas, P. N., Porter, L. D., Kelly, J. D., and Myers, J. R. 2013. Characterization of white mold disease avoidance in common bean. Eur. J. Plant Pathol. 135:525-543.

33. Papadopoulou, K., Melton, R. E., Leggett. M., Daniels, M. J., and Osbourn, A. E. 1999. Compromised disease resistance in saponin-deficient plants. Proc. Natl. Acad. Sci. USA 96:12923-12928.

34. Paxton, J. D. 1974. Phytoalexins, phenolics and other antibiotics in roots resistant to soil-borne fungi. Pages 185-192 in: Biology and Control of Soil-Borne Plant Pathogens. G. W. Bruehl, ed. American Phytopathological Society, St. Paul, MN.
35. Pedras, M. S. C. 2004. Prospects for controlling plant fungal diseases: alternatives based on chemical ecology and biotechnology. Can. J. Chem. 82:1329-1335

36. Pedras, M. S. C., and Ahiahonu, P. W. K. 2005. Metabolism and detoxification of phytoalexins and analogs by phytopathogenic fungi. Phytochemistry 66:391-411.

37. Pedras, M. S. C., Chumala, P. B., and Suchy, M. 2003. Phytoalexins from Thlaspi arvense, a wild crucifer resistant to virulent Leptosphaeria maculans: structures, syntheses and antifungal activity. Phytochemistry 64:949-956.

38. Pedras, M. S. C., Jha, M., and Ahiahonu, P. W. K. 2003. The synthesis and biosynthesis of phytoalexins produced by cruciferous plants. Curr. Org. Chem. 7:1635-1647.

39. Shanner, G., and Finney, R. E. 1977. The effect of nitrogen fertilization on the expression of slow mildewing resistance in Knox wheat. Phytopathology 67:1051-1056.

40. Thomzik, J. E., Stenzel, K., Stocker, R., Schreier, P. H., Hain, R., and Stahl, D. J. 1997. Synthesis of a grapevine phytoalexin in transgenic tomatoes (Lycopersicon esculentum Mill.) conditions resistance against Phytophthora infestans. Physiol. Mol. Plant. Pathol. 51:265-278.

41. Vuong, T., Diers, B. W., and Hartman, G. L. 2008. Identification of QTL for resistance to Sclerotinia stem rot (Sclerotinia sclerotiorum) in plant introduction 194639. Crop Sci. 48:2209-2214.

42. Vuong, T. D., Hoffman, D. D., Diers, B. W., Miller, J. F., Steadman, J. R., and Hartman, G. L. 2004. Evaluation of soybean, dry bean, and sunflower for resistance to Sclerotinia sclerotiorum. Crop Sci. 44:777-783.

43. Yu, C. K. Y., Lam, C. N. W., Springob, K., Schmidt, J., Chu, I. K., and Lo, C. 2006. Constitutive accumulation of cis-piceid in transgenic Arabidopsis overexpressing a Sorghum stilbene synthase gene. Plant Cell Physiol. 47:1017-1021.

44. Yuan, J., Njiti, V. N., Meksem, K., Iqbal, M. J., Triwitayakorn, K., Kassem, M. A., Davis, G. T., Schmidt, M. E., and Lightfoot, D. A. 2002. Quantitative trait loci in two soybean recombinant inbred line populations segregating for yield and disease resistance. Crop Sci. 42:271-277. 